\title{
Nociceptive Sensitization by Activation of Protease-Activated Receptor 2 in a Rat Model of Incisional Pain
}

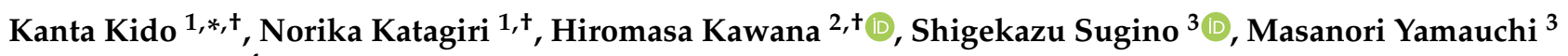 \\ and Eiji Masaki ${ }^{4}$ \\ 1 Department of Anesthesiology, Kanagawa Dental University Hospital, Yokosuka, Kanagawa 2388570, Japan; \\ katagiri@kdu.ac.jp \\ 2 Department of Oral and Maxillofacial Implantology, Kanagawa Dental University Hospital, Yokosuka, \\ Kanagawa 2388570, Japan; kawana@kdu.ac.jp \\ 3 Department of Anesthesiology and Perioperative Medicine, Tohoku University School of Medicine, Sendai, \\ Miyagi 9808575, Japan; sugino@ruby.ocn.ne.jp (S.S.); yamauchi@med.tohoku.ac.jp (M.Y.) \\ 4 Department of Anesthesiology, International University of Health and Welfare Hospital, Nasushiobara, \\ Tochigi 3292763, Japan; ejmasaki@gmail.com \\ * Correspondence: kido@kdu.ac.jp \\ + These authors contributed equally to this work and are considered co-first authors.
}

Citation: Kido, K.; Katagiri, N.; Kawana, H.; Sugino, S.; Yamauchi, M.; Masaki, E. Nociceptive Sensitization by Activation of Protease-Activated Receptor 2 in a Rat Model of Incisional Pain. Brain Sci. 2021, 11, 144. https://doi.org/10.3390/ brainsci11020144

Received: 21 December 2020

Accepted: 18 January 2021

Published: 22 January 2021

Publisher's Note: MDPI stays neutral with regard to jurisdictional claims in published maps and institutional affiliations.

Copyright: (c) 2021 by the authors. Licensee MDPI, Basel, Switzerland. This article is an open access article distributed under the terms and conditions of the Creative Commons Attribution (CC BY) license (https:/ / creativecommons.org/licenses/by/ $4.0 /)$.

\begin{abstract}
Postoperative pain and consequent inflammatory responses after tissue incision adversely affects many surgical patients due to complicated mechanisms. In this study, we examined whether activation of protease-activated receptor 2 (PAR-2), which is stimulated by tryptase from mast cells, elicits nociception and whether the PAR-2 antagonist could reduce incisional nociceptive responses in vivo and in vitro. The effects of a selective PAR-2 antagonist, N3-methylbutyryl-N-6aminohexanoyl-piperazine (ENMD-1068), pretreatment on pain behaviors were assessed after plantar incision in rats. The effects of a PAR-2 agonist, SLIGRL-NH $\mathrm{N}_{2}$, on nociception was assessed after the injection into the hind paw. Furthermore, the responses of $\mathrm{C}$-mechanosensitive nociceptors to the PAR-2 agonist were observed using an in vitro skin-nerve preparation as well. Intraplantar injection of SLIGRL-NH$H_{2}$ elicited spontaneous nociceptive behavior and hyperalgesia. Local administration of ENMD-1068 suppressed guarding behaviors, mechanical and heat hyperalgesia only within the first few hours after incision. SLIGRL- $\mathrm{NH}_{2}$ caused ongoing activity in $47 \%$ of C-mechanonociceptors in vitro. This study suggests that PAR-2 may support early nociception after incision by direct or indirect sensitization of C-fibers in rats. Moreover, PAR-2 may play a regulatory role in the early period of postoperative pain together with other co-factors to that contribute to postoperative pain.
\end{abstract}

Keywords: postoperative pain; protease-activated receptor 2; PAR-2; tryptase; mast cell

\section{Introduction}

Postoperative pain is caused by multiple processes including inflammatory, neuropathic and ischemic components [1-4]. Multiple analgesic methods and drugs have been applied to reduce postoperative pain [5,6]; nevertheless, these management strategies do not always improve the pain due to their undesirable adverse effects [6]. Various mechanisms of postoperative pain have been investigated, including excitation, sensitization and inhibition of the peripheral and central nervous system $[1,7,8]$. In peripheral tissue, many inflammatory mediators from immune cells or resident cells involving neutrophils, platelets, macrophages and mast cells, contribute to peripheral nociceptive sensitization after tissue injury $[9,10]$.

Mast cells are one of the resident immune cells in connective tissue. They play crucial roles in the early phase of immune responses and they are called "gatekeepers" [11], because they mainly reside near the nerve fibers in the subcutaneous tissue [12]. Mast cells release a variety of mediators which produce nociception on primary afferent neurons by 
binding to receptors which are expressed on the afferents [11,13-15]. A few recent studies have investigated the association between mast cells and postoperative pain. Oliveira et al. described that preventing degranulation of mast cells reduced spontaneous pain and mechanical allodynia after plantar incision in mice [16,17], and we also previously reported that mast cell stabilization by cromoglycate promoted antinociceptive effects in mice [18]. However, the antagonism of typical mediators like serotonin and histamine has shown weak efficacy in a surgery-induced pain in rodents $[16,19,20]$, suggesting that other mediators could participate in the pain process.

When mast cells degranulate and release tryptase, this produces nociception by binding to the protease activated receptor-2 (PAR-2) on primary afferents [11,13]. Recent studies have shown that the activation of PAR-2 is related to some painful diseases including arthritis and inflammatory bowel disease [21-23]. PAR-2 is one of the G protein-coupled receptors which is expressed on primary sensory neurons, and the agonist triggers release of substance $P$ and calcitonin gene-related peptide (CGRP) from peripheral nerve ending [24]. However, the part of PAR-2 activation in painful processes is still not understood. Only one study examined the effects of PAR-2 antagonist pretreatment on postoperative pain in mice [17]. In the study, antagonism of PAR-2 produced a short-lasting effect in preventing postoperative pain in vivo, and surgery increased tryptase activity in tissue perfusates, suggesting that tryptase released from mast cell was a candidate to activate PAR-2 on nociceptive terminals and contributed to nociception caused by surgery. However, little is known about the effects of PAR-2 activation on nociceptors. In this study, we examined rats and we tested whether the selective PAR-2 antagonist N3-methylbutyryl-N-6-aminohexanoyl-piperazine (ENMD-1068) could reduce incisional nociception, and whether the injection of a PAR-2 agonist SLIGRL-NH $\mathrm{N}_{2}$ elicited pain in vivo. We also examined whether the agonist activates mechanosensitive primary afferents in an in vitro skin-nerve preparation.

\section{Materials and Methods}

\subsection{General}

All experimental procedures were reviewed and approved by the Committee of Ethics on Animal Experiments of Kanagawa Dental University (protocol No. 18-035) and Institutional Laboratory Animal Care and Use Committee of Tohoku University (protocol No. 2015-009). Adult male Sprague-Dawley rats (250-300 g; SLC, Hamamatsu, Japan) were used in this study. Rats were housed under a 12-h light-dark schedule. Food and water were available ad libitum.

\subsection{Drug Preparation}

Selective PAR-2 antagonist, N3-methylbutyryl-N-6-aminohexanoyl-piperazine (ENMD1068) was purchased from Enzo Life Sciences (Farmingdale, NY, USA) and solved in phosphate buffered saline (PBS). PAR-2-activating peptide (PAR-2-AP, SLIGRL-NH $\mathrm{N}_{2}$ ), corresponding to the tethered ligand of rat PAR-2, was obtained from Sigma-Aldrich Japan (Tokyo, Japan).

\subsection{Intraplantar Drug Administration}

To investigate the effects of local subcutaneous injection of PAR-2 antagonist on the incisional model, injection (ENMD-1068; $10 \mathrm{mM}, 100 \mu \mathrm{L}$ ) was performed $30 \mathrm{~min}$ before paw incision using a microsyringe with a 30-gauge needle and under light sevoflurane anesthesia. The control group received $100 \mu \mathrm{L}$ PBS in the same manner. Guarding behavior and responses to thermal and mechanical stimulation were measured after plantar incision. The PBS solution was used as the control.

In a separate series of experiments, PAR-2-activating peptide (SLIGRL-NH $\mathrm{NH}_{2} ; 1 \mathrm{mM}$, $100 \mu \mathrm{L}$ ) was injected into the plantar surface of the hind paw subcutaneously using a 30 -gauge needle and a microsyringe in a total volume of $100 \mu \mathrm{L}$. For the control group, PBS was injected in the same manner. To assess spontaneous pain behaviors, drugs were injected into the middle of the tori of the paw using gentle restraint without anesthesia. 
Spontaneous pain and responses to heat and mechanical stimuli were measured after injection. The investigator performing the behavioral data was blinded to drug.

\subsection{Plantar Hind Paw Incision}

The surgery was performed as described previously [25]. Briefly, rats were anesthetized with 3 to $4 \%$ sevoflurane in air via a nose cone. A 10-mm longitudinal incision was made with a \#11 surgical blade through the skin, fascia, and the plantar flexor digitorum brevis muscle. Blunt-curved forceps were then inserted through the incision into the muscle to further divide and retract the muscle. The wound was then closed with two subcutaneous mattress sutures with 5-0 nylon on a P-1 needle (Ethicon, USA) and covered with 3\% tetracycline ointment from Sun pharma Japan (Tokyo, Japan). The control sham-operated rats underwent anesthesia and sterile preparation and no incision.

\subsection{Behavioral Studies}

\subsubsection{Spontaneous pain behavior}

After injection of PAR-2-AP or other solutions, rats were immediately returned into the box on the mesh floor and the ipsilateral hind paw was observed for $30 \mathrm{~min}$. Data were collected in 5-min bins after PAR-2-AP injection. Time spent shaking, licking, lifting and biting the hind paw was recorded.

\subsubsection{Guarding Behavior}

A pain score was determined to evaluate guarding pain behaviors as described previously [26]. In brief, rats were acclimated to the testing environment for two or three hours per day three days before testing. A baseline test was then performed one day before surgery. To perform guarding behavior testing, rats were individually placed into a clear plastic cage top $(21 \times 26 \times 16 \mathrm{~cm})$ on a small plastic mesh floor (grid $8 \times 8 \mathrm{~mm}$ ). Both ipsilateral and contralateral hind paws of each animal were closely observed during a one-minute period repeated for every five minutes for one hour. A guarding pain score of 0,1 or 2 was given, depending on the position in which each paw was found during one minute. A score of 0 was recorded when full weight bearing with the incised region was blanched or distorted by the mesh, 1 was recorded when the wound area just touched the mesh without blanching or distortion and 2 for the wound area completely off of the mesh surface. The sum of the 12 scores (0-24) recorded during one hour for each paw was obtained.

\subsubsection{Heat Sensitivity}

Each rat was placed on a glass floor covered with a clear plastic cage top $(21 \times 26 \times$ $16 \mathrm{~cm}$ ) and acclimated. Heat withdrawal latencies were assessed by applying a focused radiant heat source (IITC-390 Plantar Test Analgesia Meter, IITCR Life Science Instruments, Woodland Hills, CA, USA) underneath a glass floor on the middle of the incised or injected area. The intensity of the heat was set to produce withdrawal latency in control rats of $10-15 \mathrm{~s}$. Each rat was tested three times, at an interval of $10 \mathrm{~min}$. The latency to evoke a withdrawal was determined with a cut-off value of $20 \mathrm{~s}$. The average of three trials was used to obtain paw withdrawal latency.

\subsubsection{Mechanosensitivity}

Each rat was individually placed on a plastic mesh floor covered with a clear plastic cage top $(21 \times 26 \times 16 \mathrm{~cm})$ and allowed to acclimate. Withdrawal response to punctate mechanical stimulation was determined using calibrated mono filaments (Touch Test Sensory Evaluator, Stoelting, IL, USA) applied underneath the cage through to an area adjacent to the wound or the middle of tori of the paw of the injected drug. Each filament was applied once starting with $10 \mathrm{mN}$ and continuing until a withdrawal response occurred or $250 \mathrm{mN}$ was reached. When no response to the $250 \mathrm{mN}$ filaments was observed, the next filament $522 \mathrm{mN}$ was recorded. Each rat was tested three times with at least a 5-min interval 
between withdrawal responses. The lowest force from the three tests producing a response was determined as the withdrawal threshold [27].

\subsection{In Vitro Single Fiber Recordings \\ 2.6.1. Preparation}

The rat in vitro skin-nerve preparation has been previously described [28]. Briefly, the rats were euthanized with carbon dioxide, and medial and lateral plantar nerves and their innervated glabrous hind paw skin were dissected and removed. The isolated preparation was immediately placed in an organ bath, which was continuously superfused with synthetic interstitial fluid (SIF; $107 \mathrm{mM}$ of $\mathrm{NaCl}, 26.2 \mathrm{mM}$ of $\mathrm{NaHCO}_{3}, 9.64 \mathrm{mM}$ of sodium gluconate, $5.5 \mathrm{mM}$ of glucose, $7.6 \mathrm{mM}$ of sucrose, $3.48 \mathrm{mM}$ of $\mathrm{KCl}, 1.67 \mathrm{mM}$ of $\mathrm{NaH}_{2} \mathrm{PO}_{4}$, $1.53 \mathrm{mM}$ of $\mathrm{CaCl}_{2}$, and $0.69 \mathrm{mM}$ of $\mathrm{MgSO}_{4}$ ), which had been oxygenated with a mixture of $95 \% \mathrm{O}_{2}$ and $5 \% \mathrm{CO}_{2}$. The temperature of the solution was maintained at $32{ }^{\circ} \mathrm{C} \pm 0.5^{\circ} \mathrm{C}$. The skin was placed epidermal side down and the plantar nerve was drawn through a small hole to the recording chamber, which was filled with paraffin oil. The nerve was placed on a mirror, desheathed, and filaments were repeatedly teased using sharpened forceps and placed on a platinum electrode under microscopic view, until single-unit activity could be recorded. Neural activity was amplified (DAM50, Harvard Apparatus, Holliston, MA), filtered, and displayed using standard techniques. Amplified signals were led to an oscilloscope and an audiomonitor and fed into a PC computer via a data acquisition system (spike2/CED1401 program, Cambridge Electronic Design, Cambridge, UK).

\subsubsection{Identification of Afferents}

We focused on the mechanosensitive C-fiber nociceptors in this study. Receptive fields were found by probing with a fire polished glass rod in the subdermal side of skin. Only units that were clearly distinguished (signal-to-noise ratio greater than 2:1) were further studied.

\subsubsection{Ongoing Activity}

The receptive field was identified using a hollow metal cylinder with silicone grease to prevent leakage from the bath into the receptive field. After a 3-min baseline recording, the SIF solution inside the ring was removed with a syringe. Then, either SLIGRL- $\mathrm{NH}_{2}$ $100 \mu \mathrm{M}$ or PBS-vehicle in-200 $\mu \mathrm{L}$ volume was applied to the receptive field and the activity was recorded for $3 \mathrm{~min}$. The afferent was considered activated by SLIGRL-NH $\mathrm{N}_{2}$ if activity of at least $0.1 \mathrm{impulse} / \mathrm{s}$ was produced if ongoing background activity was absent during baseline recording, or if ongoing activity was present an increase of at least two standard deviations greater than the ongoing background activity.

\subsubsection{Heat Stimulation}

The effect of SLIGRL-NH${ }_{2}$ on heat response was then tested using a ramp-shaped thermal stimulus to the receptive field using a feedback-controlled Peltier thermal stimulator with a circular probe (diameter: $1 \mathrm{~mm}$, intercross-2000 N, Intercross, Tokyo, Japan). After the effect of SLIGRL-NH${ }_{2}$ or PBS-vehicle on ongoing activity was complete, a computercontrolled heat ramp was delivered starting from 32 to $47^{\circ} \mathrm{C}$ over $30 \mathrm{~s}$. The peak temperature, $47^{\circ} \mathrm{C}$, was determined to avoid potential tissue damage of higher temperatures. Each fiber was examined only once with either SLIGRL- $\mathrm{NH}_{2}$ or PBS-vehicle for heat responses and tested in separate experiments for mechanical stimuli preventing heat stimuli from sensitizing the afferent responses. Action potentials were counted during the heat stimulus (for $30 \mathrm{~s}$ ) and for $20 \mathrm{~s}$ after the peak temperature. The temperature that induced the first action potential was considered as heat threshold under no background ongoing activity. If background activity was present, the threshold was defined by the temperature during heating that increased background activity at least two standard deviations greater than the baseline (10 s, $1 \mathrm{~s}$ bin). Ongoing activity of background was subtracted from total 
action potential numbers during the recording period, postulating that background activity continued during the stimulation.

\subsubsection{Mechanical Stimulation}

Mechanosensitivity was assessed using a servo force-controlled mechanical stimulator (Series 300C Dual Mode Servo System; Aurora Scientific, Aurora, Ontario, Canada). A flatended cylindrical metal probe (tip diameter, $0.7 \mathrm{~mm}$ ) attached to the tip of the stimulator arm was applied to the most sensitive point of the receptive field with no force generated.

For mechanical testing, each fiber was examined twice, once each with PBS-vehicle and subsequently with SLIGRL-NH${ }_{2}$. First, the SIF solution inside the ring was removed and PBS-vehicle solution with 200- $\mu \mathrm{L}$ volume was applied. After 2 min of PBS application, a computer-controlled ascending series of square force stimuli was applied to the receptive field (5, 10, 20, 40, 80 and $120 \mathrm{mN}$; $2 \mathrm{~s}$ duration; $60 \mathrm{~s}$ intervals). After testing PBS with application, the solution inside the ring was gently replaced with SIF solution a few times for washing out. Then, the SIF solution inside the ring was replaced with $100 \mu \mathrm{M}$ SLIGRL$\mathrm{NH}_{2}$ with $200-\mu \mathrm{L}$ volume. After 2 min of drug application, the same series of mechanical stimulations was applied to the receptive field. The mechanical force which evoked the first action potential was determined as the mechanical threshold under no background activity. If background activity was present, mechanical threshold was determined by the force that increased background activity at least two standard deviations greater than the average background for baseline recording. Ongoing activity of background was subtracted from the total number of action potentials during mechanical stimuli, postulating that background activity continued during the stimulation.

\subsubsection{Conduction Velocity}

The conduction velocity was evaluated at the end of the experiment. The conduction velocity of each fiber was determined by a bipolar electrode to induce action potentials in the afferent in the most mechanosensitive spot in the receptive filed $(0.2-1.0 \mathrm{~Hz}, 5-20 \mathrm{~V}$, 0.5-2.0 ms duration). Then the distance between the recording electrode (conduction distance) and the receptive field was divided by the latency of the action potential. Afferent fibers conducting slower than $2.5 \mathrm{~m} / \mathrm{s}$ were classified as C-fibers, those conducting between 2.5 and $24 \mathrm{~m} / \mathrm{s}$ as A $\delta$-fibers, and those conducting faster than $24 \mathrm{~m} / \mathrm{s}$ as A $\beta$-fibers. Units were classified as mechanosensitive nociceptors on the basis of their graded response throughout the innocuous and noxious range of mechanical force stimuli. Rapidly adapting fibers were excluded. A-delta fibers were also recorded. However, the number was too few to analyze (5 fibers for ongoing activities, 4 for mechanical responses and 2 for heat responses).

\subsection{Statistical Analysis}

The Kolmogorov-Smirnov test was used for testing of normality. Guarding pain score and withdrawal latency to heat data were analyzed using 2-way analysis of variance (ANOVA), followed by 1-way ANOVA and the post hoc t-test with Bonferroni correction. Spontaneous pain behaviors were analyzed using 2-way ANOVA. The differences between groups were evaluated using two-way repeated measures ANOVA followed by the post hoc Sidak's multiple comparisons test. Mechanical behavioral data were analyzed the using Friedman test. Between group differences were subsequently analyzed by the Mann-Whitney rank sum test (two groups) to compare drug at each time point vs. PBSvehicle. All behavioral data are presented as means \pm standard error of the mean (SEM). The chi-square test was performed to analyze prevalence of PAR-2-AP responsive and heat responsive units between groups for electrophysiological data. Heat response threshold and total action potentials during heat stimulation were analyzed using the Mann-Whitney rank sum test. The mechanical stimulus-response functions between groups were analyzed using two-way ANOVA with repeated measures. Data are presented as means \pm SEM. 
A $p$-value less than 0.05 was considered statistically significant. All tests were performed with GraphPad Prism software (GraphPad, San Diego, CA, USA).

\section{Results}

\subsection{Behavioral Studies}

\subsubsection{Effect of PAR-2 Agonist, SLIGRL-NH $\mathrm{N}_{2}$ on Pain Behaviors}

PBS-vehicle produced very little spontaneous pain behavior (Figure 1A). There was a greater effect after injection of $1 \mathrm{mM} 100 \mu \mathrm{L}$ of SLIGRL- $\mathrm{NH}_{2}$, which produced nociceptive behavior from $5 \mathrm{~min}$ to $10 \mathrm{~min}$ compared with PBS-vehicle $(0.6 \pm 0.2$ vs. $13.7 \pm 3.1, p<0.001$; $n=8$ per group). PBS-vehicle did not increase the mechanical responses compared with pre injection (Figure 1B). However, intraplantar injection of PAR-2-AP significantly induced mechanical hyperalgesia for one day after injection. Treatment of PBS did not decrease the withdrawal latency to radiant heat compared with pre injection (Figure 1C). Intraplantar injection of PAR-2-AP significantly induced the heat hypersensitivity compared with PBSvehicle from $30 \mathrm{~min}$ to 2 days after injection.
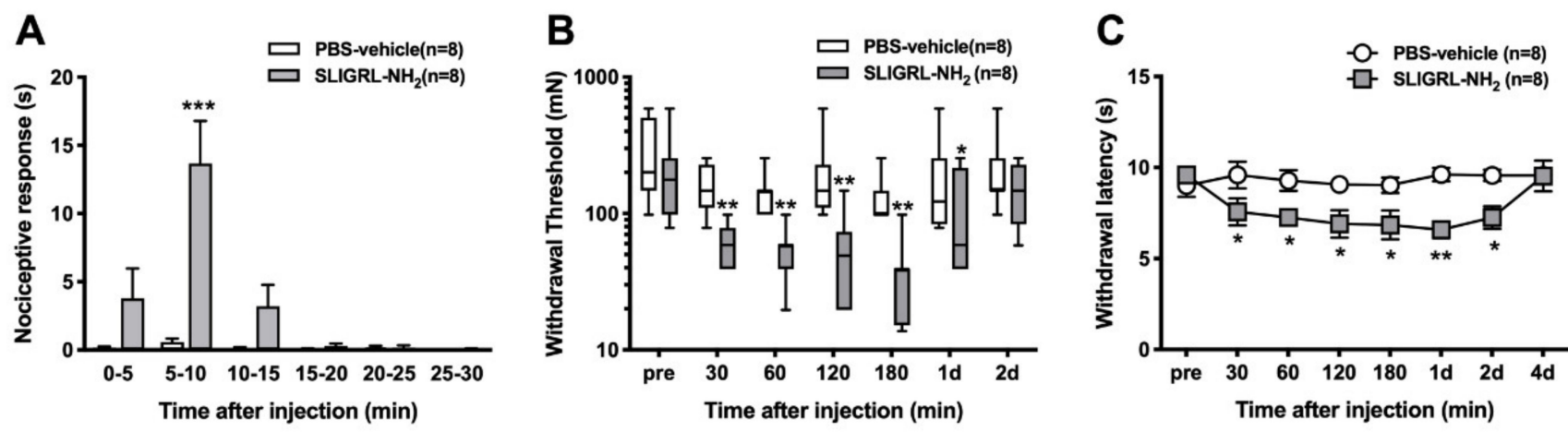

Figure 1. Effects of protease-activated receptor 2 (PAR-2) agonist SLIGRL-NH 2 on nociceptive behavior, withdrawal threshold to mechanical stimuli, and withdrawal latency to heat after injection. (A) Time course of the nociceptive behavior including shaking, licking, lifting and biting of the hind leg after intraplantar injection of SLIGRL-NH $\mathrm{N}_{2}$ or phosphate buffered saline (PBS) ( $n=8$ to both groups). Data were collected in 5-min bins from eight animals in each group. Data show the mean and standard error of the mean (SEM) of nociceptive time in 5-min bins. Statistical analysis used 2-way ANOVA with repeated measures on one factor followed by Bonferroni's post hoc test for comparing the mean of the nociceptive time at each 5-min point. (B) Withdrawal threshold to mechanical stimuli applied to the hind paw. The results are presented as the median with range, interquartile range (box) and 10th and 90th percentile for eight rats in each group ( $n=8$ to both groups). A non-parametric Friedman's test followed by Dunn's post hoc test for between group comparisons at each time point were used. (C) Withdrawal latency to heat stimuli. The results are presented as the mean and SEM. Two-way ANOVA with repeated measures on one factor followed by Bonferroni's post hoc test for comparing the mean withdrawal latency at each time point between groups ( $n=8$ to both groups) was performed. ${ }^{*} p<0.05,{ }^{* *} p<0.01,{ }^{* * *} p<0.001$ compared with the PBS-vehicle group at each time point.

\subsubsection{Effect of PAR-2 Antagonist, ENMD-1068 on Pain Behaviors after Plantar Incision}

In the group of incised rats treated with PBS-vehicle, the guarding pain score increased from one hour to two days after incision compared with baseline (Figure 2A). Pretreatment with ENMD-1068 decreased the guarding behavior by paw incision at $1 \mathrm{~h}$ compared with PBS. This was the only significant decrease in cumulative pain score $(10.6 \pm 1.3 \mathrm{vs}$. $16.9 \pm 1.0, p<0.001$, PBS; $n=8$ per group). Paw incision induced a decrease in mechanical withdrawal threshold a during the 4 days following incision as displayed in Figure 2B. Intraplantar injection of ENMD-1068 increased the mechanical withdrawal threshold from $1 \mathrm{~h}$ to $2 \mathrm{~h}$ after incision. In the PBS-treated group, the mean withdrawal latency to radiant heat decreased from sec s $14.9 \pm 0.6$ to $3.2 \pm 0.21 \mathrm{~h}$ after incision (Figure 2C). One hour after incision, ENMD-1068 increased the withdrawal latency compared to the PBS control $(5.7 \pm 0.7 ; p<0.01)$. ENMD-1068 did not affect heat withdrawal latency thereafter. 

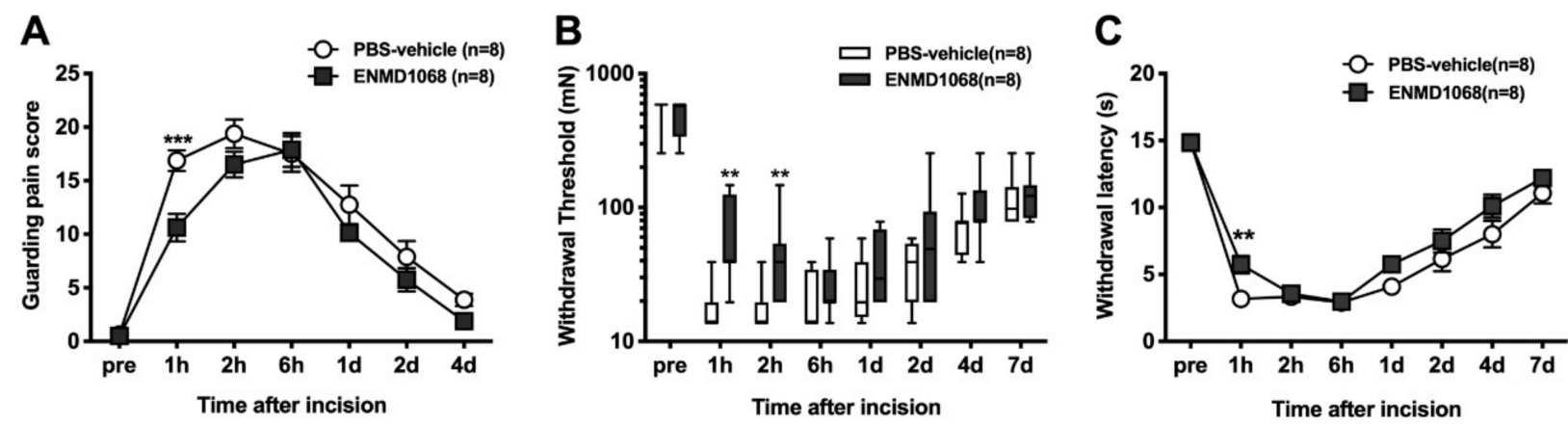

Figure 2. Effects of PAR-2 antagonist N3-methylbutyryl-N-6-aminohexanoyl-piperazine (ENMD 1068) on pain behaviors of rats after incision. (A) Guarding pain behavior. The results are presented as the mean and SEM for eight rats in each group ( $n=8$ to both groups). Statistical analysis used 2-way ANOVA with repeated measures on one factor followed by Bonferroni's post hoc t-test for comparing the mean cumulative pain score at each time point between groups. (B) Withdrawal threshold to mechanical stimuli applied to the hind paw. The results are presented as the median with interquartile range (box) and 10th and 90th percentile for eight rats in each group ( $n=8$ to both groups). A non-parametric Friedman's test followed by Dunn's post hoc test for between- group comparisons at each time point were used. (C) Withdrawal latency to heat stimuli. The results are presented as the mean and SEM. Statistical analysis used 2-way ANOVA with repeated measures on one factor followed by Bonferroni's post hoc test for comparing the mean withdrawal latency at each time point between groups ( $n=8$ to both groups). ${ }^{* *} p<0.01,{ }^{* * *} p<0.001$ compared with the vehicle group at each time point. POD = postoperative day.

\subsection{Single Fiber Recording}

PBS-vehicle did not increase the ongoing activity of C-fibers (Figure 3A,B). SLIGRL$\mathrm{NH}_{2}$ increased the ongoing activity of 10 of $21 \mathrm{C}$-mechanonociceptive fibers (47.6\%) (Figure 3D). The median increase in activity after application of SLIGRL-NH $\mathrm{N}_{2}$ was 36 $\mathrm{imp} / 3 \mathrm{~min}$ (Figure $3 \mathrm{~A}, \mathrm{C}$ ). For heat, SLIGRL- $\mathrm{NH}_{2}$ did not increase the proportion of heat responsive $C$-fibers $(15 / 20,75.0 \% ; p=0.31)$ compared to PBS-vehicle $(10 / 18,55.6 \%)$ (Figure 4A,B). SLIGRL-NH $\mathrm{NH}_{2}$ did not affect the heat threshold or the magnitude of the heat response (Figure $4 \mathrm{C}, \mathrm{D}$ ). The median mechanical thresholds of C-fibers after exposure to SLIGRL-NH $\mathrm{N}_{2}$ was lower than PBS-vehicle (20 vs. $40 \mathrm{mN} ; p<0.05$ ) (Figure 5A,B). The number of action potentials at each force was not different between SLIGRL- $\mathrm{NH}_{2}$ and PBS-vehicle (Figure 5C). SLIGRL-NH ${ }_{2}$ had also no effect on the mechanical responses of C-fibers (Figure 5A). 
A

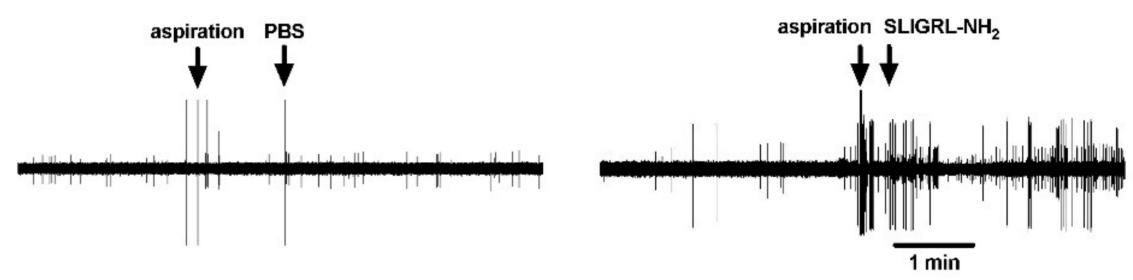

B

C

D
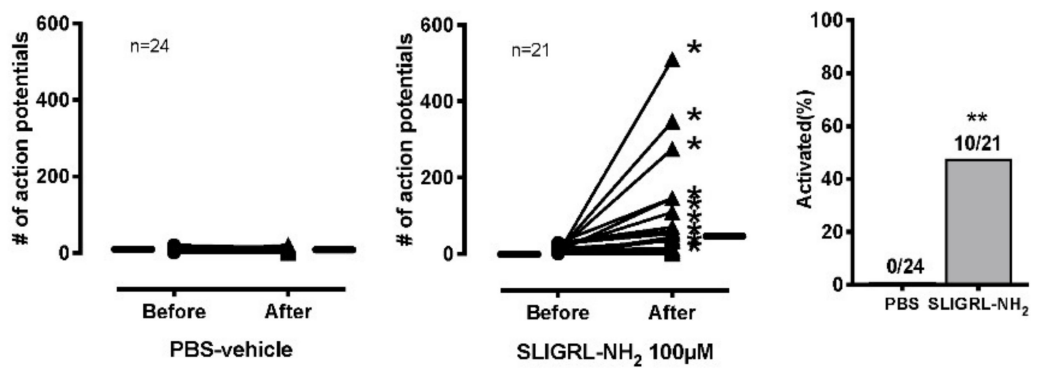

Figure 3. Effects of PAR-2 agonist SLIGRL- $\mathrm{NH}_{2}$ or vehicle on ongoing activity of mechanonociceptive C-fibers. Each fiber was tested with one application, either PBS or SLIGRL-NH2 (A) Example recordings of from 2 different $C$-fibers before and after application of PBS-vehicle or $100 \mu \mathrm{M}$ SLIGRL$\mathrm{NH}_{2}$. (B,C) Ongoing activities of C-fibers before and after exposure to $100 \mu \mathrm{M}$ SLIGRL-NH $\mathrm{N}_{2}$ or PBSvehicle. Each line on the graphs represents a single unit. Asterisks indicate activation of nociceptors by drug application. Small horizontal lines in each graph indicate median values. (D) The proportion of C-fibers responsive to application of SLIGRL-NH $\mathrm{N}_{2}$ or PBS-vehicle. A total of 24 units for PBS and 21 units for SLIGRL-NH$H_{2}$ were used.

A
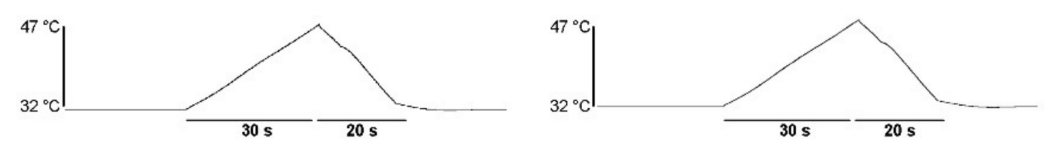

PBS

SLIGRL-NH
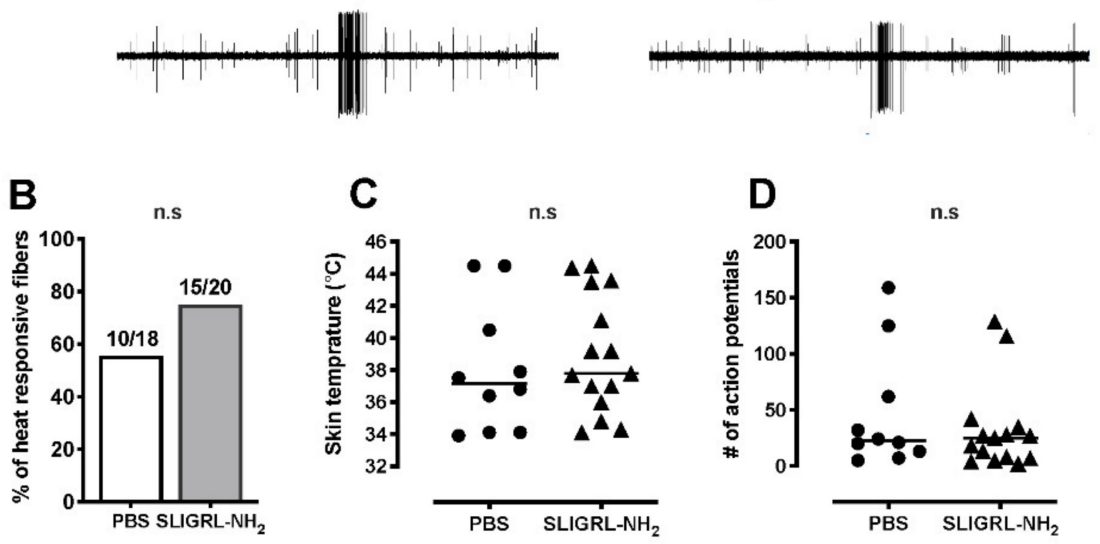

Figure 4. Effects of PAR-2 agonist SLIGRL- $\mathrm{NH}_{2}$ on heat responses of mechanonociceptive C-fibers. (A) Example recordings of C-fibers after application of either PBS-vehicle or $100 \mu \mathrm{M}$ SLIGRL-NH ${ }_{2}$. Each fiber was tested with one application, either PBS or SLIGRL-NH $\mathrm{N}_{2}$. (B) Proportion of C-fibers responsive to heat after application of SLIGRL-NH 2 or PBS-vehicle. (C,D) Heat responses of C-fibers after exposure to SLIGRL-NH ${ }_{2}$ or PBS-vehicle. Heat thresholds (C) and total action potentials for 50 s during heat application (D). Each symbol represents a single unit. 
A
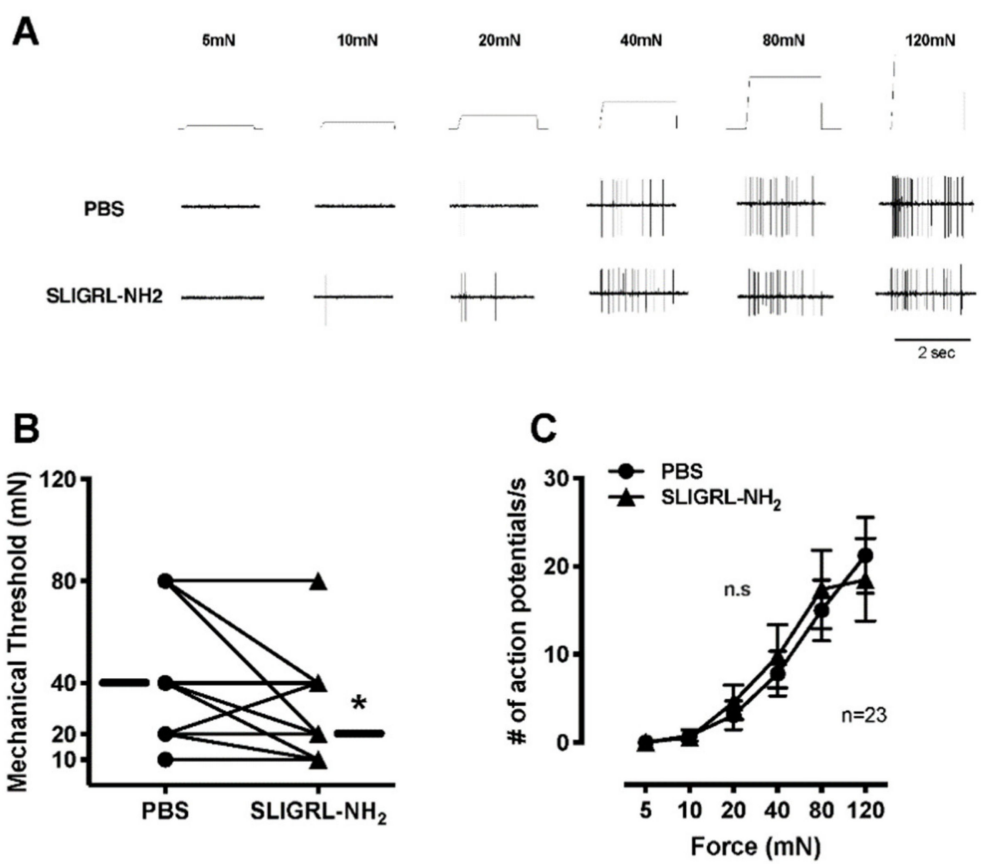

Figure 5. Effects of PAR-2 agonist SLIGRL-NH $\mathrm{N}_{2}$ on mechanical responses of mechanonociceptive C- fibers. Each fiber was tested with application of PBS followed by SLIGRL-NH $\mathrm{NH}_{2}$. (A) Example recordings of C-fibers after application of PBS-vehicle and $100 \mu \mathrm{M}$ SLIGRL-NH $\mathrm{NH}_{2}$. (B) Mechanical thresholds of C-fibers after application of PBS-vehicle and SLIGRL-NH $\mathrm{N}_{2}$. Each line represents a single unit. The horizontal lines in each graph indicate median values. (C) Mechanical stimulus response function of $\mathrm{C}$-fibers after application PBS-vehicle and SLIGRL-NH 2 . Data are presented as means \pm SEM. ${ }^{*} p<0.05$.

\section{Discussion}

We tested whether protease-activated receptor 2 (PAR-2), influenced incisional nociception in vivo in rats. In addition, we examined the ability of the PAR-2 agonist SLIGRL- $\mathrm{NH}_{2}$ to activate or sensitize primary afferent in the in vitro skin-nerve preparation. The main findings of this study are that (i) activation of PAR-2 using an agonist, SLIGRL-NH ${ }_{2}$ induced nociceptive pain behavior and increased heat and mechanical responsiveness, (ii) blockade of PAR-2 using a selective antagonist, ENMD-1068, reduced incision-induced guarding pain behavior, mechanical and heat hypersensitivity, (iii) SLIGRL- $\mathrm{NH}_{2}$ caused C-nociceptor activation but did not affect the heat and mechanical responsiveness in the in vitro skinnerve preparation. These results indicate that the activation of PAR-2 by incision induced spontaneous nociceptive effects, alongside mechanical and heat hypersensitivity; however, the effects of PAR-2 activation alone were limited in vitro. Taken together, other co-factors could be needed to contribute to sustained activation and sensitization of nociceptors.

Previously, it was demonstrated that intraplantar administration of cromoglycate, a mast cell stabilizer, reduced the pain responses expressed as guarding pain and mechanical hyperalgesia in a mouse postoperative pain model [18]. Thus, stabilization of mast cells at an operation site might reduce postoperative pain caused by various mediators from the mast cell degranulation.

Moreover, in work by others, the PAR-2 agonist, SLIGRL- $\mathrm{NH}_{2}$, induced nociceptive pain behaviors immediately after the injection and significantly increased mechanical and heat hyperalgesia within a few days after administration in mice. To our knowledge, only one study examined a role for PAR-2 in non-evoked pain [29]. Tillu et al. described that intraplantar injection of PAR-2 agonist in mice caused an increase in spontaneous pain score, and the duration of the pain state was less compared with mechanical hypersensitivity. They concluded that PAR-2 agonist directly evoked spontaneous pain behavior [29]. 
Studies by others support a role for PAR-2 in nociception. Vergnolle et al. described that proteinases and selective PAR-2 agonists stimulated nociceptive neurons and caused hyperalgesia to both heat and mechanical stimuli in mice [30]. They described that PAR-2 was expressed on dorsal root ganglion (DRG) neurons which also expressed CGRP and Substance P, and activation of PAR-2 directly signaled to the neurons and stimulated the release of both CGRP and Substance P from C-fibers in peripheral tissues and in the spinal cord. In our in vitro study, a PAR-2 agonist directly induced ongoing activity of C-fiber but had little effect on the heat and mechanical responses of the primary afferents. These results, taken together, indicate that PAR-2 activation might be one component of early postoperative spontaneous ongoing pain and promotes release of the peptides including Substance P or CGRP from DRG and spinal cord, which lead to heat and mechanical hyperalgesia. Furthermore, other pronociceptive mediators or other receptors' activation, including TRPV1, [31] affected by PAR-2 activation, could play crucial roles in sustaining or potentiating pain after surgery. In fact, protons, prostaglandins, interleukins and nerve growth factor (NGF) have been demonstrated to be released later after the incision [32-36]. PAR-2 activated by mast cell tryptase could be activated early in postoperative pain.

In this study, blockade of PAR-2 using a selective antagonist, ENMD-1068 briefly decreased incision-induced guarding pain, mechanical and heat hypersensitivity, highlighting the contribution of peripheral PAR-2 to early incisional pain (Figure 2). Preventing mast cells from degranulating (with Compound 48/80 prior to incision or pretreatment of mast cell stabilizer) markedly reduced postoperative hyperalgesia in mice $[16,18]$. Meanwhile, antagonism of histamine or serotonin receptors only partially reduced the postoperative mechanical hyperalgesia and non-evoked pain in mice [16]. Therefore, it was considered that other mediators from mast cells must be involved. Only one study focused on the effects of PAR-2 antagonist on postoperative pain in a preclinical study [17]. Oliveira et al. described that pretreatment with the selective PAR-2 antagonist ENMD-1068, and the selective tryptase inhibitor gabexate, reduced postoperative mechanical hyperalgesia and spontaneous nociception in a mouse model of postoperative pain.

Although pretreatment with ENMD-1068 reduced guarding pain score, mechanical and heat hyperalgesia after plantar incision in our study, the efficacy might be limited to the early period after surgery. One of the reasons may be that the activity of tryptase (or other mediators from mast cells as well) was detected within $10 \mathrm{~min}$ but not 30 or $60 \mathrm{~min}$ after plantar surgery [17]. In fact, Oliveira et al. examined the effect of posttreatment with ENMD-1068, PAR-2 antagonist, on mechanical hyperalgesia after plantar incision. They showed that the antagonist did not alter the intensity of postoperative nociception [17]. Furthermore, it was considered that the PAR-2-induced pain pathway after incision is not sufficient to block pain related behaviors, and that other mediators contribute [1,37].

In our study, SLIGRL-NH ${ }_{2}$, a PAR-2 agonist caused ongoing activity which was increased in $47 \%$ of C-mechanonociceptors in vitro skin-nerve preparation but had little effect on the heat and mechanical responsiveness of the afferents. Previous studies have shown that PAR-2 was expressed by approximately $60 \%$ of neurons in the dorsal root ganglia, where it was found in small-diameter, nociceptive neurons containing Substance $\mathrm{P}$ and CGRP [24,38]. Supporting the behavioral tests in this study, PAR-2 activation directly excited the C-mechanosensitive nociceptors, however, did not sensitize the primary nociceptors to heat and mechanical stimuli. These results suggested that (i) the nociception of the primary afferents by PAR-2 activation resulting in spontaneous activity might contribute to heat and mechanical responses via central sensitization or (ii) the nociception by PAR-2 activation might require other circulating molecules that affected sensory neuron excitability, because these might have been "washed out" in our in vitro recording conditions.

Recently, the cooperative interaction between PAR-2 activation and transient receptor potential (TRP) ion channels including TRPV1, TRPV4 and TRPA1 has been clarified in nociceptive signaling and pain [39-41]. PAR-2 is highly co-expressed with TRPV1 receptors in the DRG neurons [31], and PAR-2 activation leads to TRPV1 sensitization to endogenous 
agonist [42,43]. PAR-2 activation might be a trigger which enhanced TRP channels or other receptors leading to the nociception of the primary afferents [29].

There were several limitations to this study. First, we did not examine the dose dependency of the effect of PAR-2 antagonist, ENMD 1068, and agonist, SLIGRL-NH ${ }_{2}$. Both optimal doses were based on previous studies [17,30]. In the study by Oliveira et al. [17], intraplantar administration of $100 \mathrm{nmol} / 20 \mu \mathrm{L}$ ENMD-1068 (33 $\mu \mathrm{g} / \mathrm{paw} \fallingdotseq 1 \mu \mathrm{g} / \mathrm{g}$ body weight) was effective for blockade of PAR-2 in mice and hence $10 \mathrm{mM} / 100 \mu \mathrm{L}$ ENMD-1068 $(330 \mu \mathrm{g} / \mathrm{paw} \fallingdotseq 1 \mu \mathrm{g} / \mathrm{g}$ body weight) was injected intraplantarly in rats in our study. Similarly, in the study by Vergnolle et al. [30], intraplantar administration of $1 \sim 10 \mu \mathrm{g} / \mathrm{paw}$ SLIGRL- $\mathrm{NH}_{2}$ was effective to induce hyperalgesia in mice, and hence $1 \mathrm{mM} / 100 \mu \mathrm{L}$ $(77.1 \mu \mathrm{g} / \mathrm{paw})$ was injected in rats. Second, mechanosensitive-A $\delta$ fibers were not assessed, because insufficient numbers of A $\delta$ were collected for analysis. However, PAR-2 was expressed by approximately $60 \%$ of neurons in the dorsal root ganglia, where it was found in small-diameter, mostly nociceptive C-fibers containing Substance P and CGRP [30]. Therefore, a focus on C-fibers is warranted.

\section{Conclusions}

Our results suggest that PAR-2 may support early nociception after incision by sensitization of C-nociceptors in rats. Moreover, PAR-2 may contribute to the development of postoperative pain together with other inflammatory mediators or channel activation. Further studies are needed to determine other co-factors which enhance and sustain postoperative pain.

Author Contributions: Conceptualization, K.K.; methodology, N.K., H.K., S.S.; validation, H.K. and E.M.; formal analysis, K.K. and E.M.; investigation, K.K. and S.S.; data curation, K.K.; writingoriginal draft preparation, K.K., N.K. and H.K.; writing-review and editing, M.Y. and E.M.; visualization, N.K.; supervision, M.Y. and E.M.; project administration, H.K.; funding acquisition, K.K. and M.Y. All authors have read and agreed to the published version of the manuscript.

Funding: This research was funded in part by Grants-in-Aid for Scientific Research (B) and (C) from the Japan Society for the Promotion of Science to K.K. (No. 19147263) and M.Y. (No. 17914471).

Institutional Review Board Statement: The study was conducted according to the guidelines of the Declaration of Helsinki, and approved by the Committee of Ethics on Animal Experiments of Kanagawa Dental University (protocol No. 18-035; ) and Institutional Laboratory Animal Care and Use Committee of Tohoku University (protocol No. 2015-009).

Informed Consent Statement: Not available.

Data Availability Statement: The data presented in this study are available on request from the corresponding author.

Acknowledgments: The authors wish to thank Timothy J. Brennan, Emeritus Professor of University of Iowa, for his help in interpreting the significance of the results of this study and revising the manuscript.

Conflicts of Interest: The authors declare no conflict of interest.

\section{References}

1. Pogatzki-Zahn, E.M.; Segelcke, D.; Schug, S.A. Postoperative pain-from mechanisms to treatment. Pain Rep. 2017, 2, e588. [CrossRef]

2. Kehlet, H.; Jensen, T.S.; Woolf, C.J. Persistent postsurgical pain: Risk factors and prevention. Lancet 2006, 367, 1618-1625. [CrossRef]

3. Kido, K.; Gautam, M.; Benson, C.J.; Gu, H.; Brennan, T.J. Effect of deep tissue incision on $\mathrm{pH}$ responses of afferent fibers and dorsal root ganglia innervating muscle. Anesthesiology 2013, 119, 1186-1197. [CrossRef] [PubMed]

4. Brennan, T.J. Pathophysiology of postoperative pain. Pain 2011, 152, S33-S40. [CrossRef] [PubMed]

5. Moiniche, S.; Dahl, J.B.; Kehlet, H. A qualitative and quantitative systematic review of preemptive analgesia for postoperative pain relief: the role of timing of analgesia. Anesthesiology 2002, 96, 725-741. [CrossRef] [PubMed]

6. Wall and Melzack's Textbook of Pain; McMahon, S.B.; Klotzenburg, M. (Eds.) Elsevier: Philadelphia, PA, USA, 2006; pp. 635-651. 
7. Vadivelu, N.; Mitra, S.; Narayan, D. Recent advances in postoperative pain management. Yale J. Biol. Med. 2010, 83, 11-25. [PubMed]

8. Eisenach, J.C.; Brennan, T.J. Pain after surgery. Pain 2018, 159, 1010-1011. [CrossRef]

9. Basbaum, A.I.; Bautista, D.M.; Scherrer, G.; Julius, D. Cellular and molecular mechanisms of pain. Cell 2009, 139, 267-284. [CrossRef]

10. Marchand, F.; Perretti, M.; McMahon, S.B. Role of the immune system in chronic pain. Nat. Rev. Neurosci. 2005, 6, 521-532. [CrossRef]

11. Chatterjea, D.; Martinov, T. Mast cells: Versatile gatekeepers of pain. Mol. Immunol. 2015, 63, 38-44. [CrossRef]

12. Brown, J.M.; Wilson, T.M.; Metcalfe, D.D. The mast cell and allergic diseases: Role in pathogenesis and implications for therapy. Clin. Exp. Allergy 2008, 38, 4-18. [CrossRef] [PubMed]

13. Aich, A.; Afrin, L.B.; Gupta, K. Mast Cell-Mediated Mechanisms of Nociception. Int. J. Mol. Sci. 2015, 16, 29069-29092. [CrossRef] [PubMed]

14. Kuner, R. Central mechanisms of pathological pain. Nat. Med. 2010, 16, 1258-1266. [CrossRef] [PubMed]

15. Pinho-Ribeiro, F.A.; Verri, W.A., Jr.; Chiu, I.M. Nociceptor Sensory Neuron-Immune Interactions in Pain and Inflammation. Trends Immunol. 2017, 38, 5-19. [CrossRef] [PubMed]

16. Oliveira, S.M.; Drewes, C.C.; Silva, C.R.; Trevisan, G.; Boschen, S.L.; Moreira, C.G.; de Almeida Cabrini, D.; Da Cunha, C.; Ferreira, J. Involvement of mast cells in a mouse model of postoperative pain. Eur. J. Pharmacol. 2011, 672, 88-95. [CrossRef]

17. Oliveira, S.M.; Silva, C.R.; Ferreira, J. Critical role of protease-activated receptor 2 activation by mast cell tryptase in the development of postoperative pain. Anesthesiology 2013, 118, 679-690. [CrossRef]

18. Yasuda, M.; Kido, K.; Ohtani, N.; Masaki, E. Mast cell stabilization promotes antinociceptive effects in a mouse model of postoperative pain. J. Pain Res. 2013, 6, 161-166. [CrossRef]

19. Medhurst, S.J.; Collins, S.D.; Billinton, A.; Bingham, S.; Dalziel, R.G.; Brass, A.; Roberts, J.C.; Medhurst, A.D.; Chessell, I.P. Novel histamine H3 receptor antagonists GSK189254 and GSK334429 are efficacious in surgically-induced and virally-induced rat models of neuropathic pain. Pain 2008, 138, 61-69. [CrossRef]

20. Dong, R.; Yu, B.; Chen, L.; Yu, W. The 5-HT2A receptor potassium-chloride cotransporter 2 signaling pathway in a rat incision pain model. Exp. Ther. Med. 2016, 12, 3583-3588. [CrossRef]

21. Ramachandran, R.; Noorbakhsh, F.; Defea, K.; Hollenberg, M.D. Targeting proteinase-activated receptors: Therapeutic potential and challenges. Nat. Rev. Drug Discov. 2012, 11, 69-86. [CrossRef]

22. Cenac, N. Protease-activated receptors as therapeutic targets in visceral pain. Curr. Neuropharmacol. 2013, 11, 598-605. [CrossRef] [PubMed]

23. Vergnolle, N. Protease-activated receptors as drug targets in inflammation and pain. Pharmacol. Ther. 2009, 123, 292-309. [CrossRef] [PubMed]

24. Steinhoff, M.; Vergnolle, N.; Young, S.H.; Tognetto, M.; Amadesi, S.; Ennes, H.S.; Trevisani, M.; Hollenberg, M.D.; Wallace, J.L.; Caughey, G.H.; et al. Agonists of proteinase-activated receptor 2 induce inflammation by a neurogenic mechanism. Nat. Med. 2000, 6, 151-158. [CrossRef] [PubMed]

25. Brennan, T.J.; Vandermeulen, E.P.; Gebhart, G.F. Characterization of a rat model of incisional pain. Pain 1996, 64, 493-501. [CrossRef]

26. Zahn, P.K.; Gysbers, D.; Brennan, T.J. Effect of systemic and intrathecal morphine in a rat model of postoperative pain. Anesthesiology 1997, 86, 1066-1077. [CrossRef]

27. Zahn, P.K.; Brennan, T.J. Primary and secondary hyperalgesia in a rat model for human postoperative pain. Anesthesiology 1999, 90, 863-872. [CrossRef]

28. Banik, R.K.; Brennan, T.J. Spontaneous discharge and increased heat sensitivity of rat C-fiber nociceptors are present in vitro after plantar incision. Pain 2004, 112, 204-213. [CrossRef]

29. Tillu, D.V.; Hassler, S.N.; Burgos-Vega, C.C.; Quinn, T.L.; Sorge, R.E.; Dussor, G.; Boitano, S.; Vagner, J.; Price, T.J. Protease-activated receptor 2 activation is sufficient to induce the transition to a chronic pain state. Pain 2015, 156, 859-867. [CrossRef]

30. Vergnolle, N.; Bunnett, N.W.; Sharkey, K.A.; Brussee, V.; Compton, S.J.; Grady, E.F.; Cirino, G.; Gerard, N.; Basbaum, A.I.; Andrade-Gordon, P.; et al. Proteinase-activated receptor-2 and hyperalgesia: A novel pain pathway. Nat. Med. 2001, 7, 821-826. [CrossRef]

31. Amadesi, S.; Nie, J.; Vergnolle, N.; Cottrell, G.S.; Grady, E.F.; Trevisani, M.; Manni, C.; Geppetti, P.; McRoberts, J.A.; Ennes, H.; et al. Protease-activated receptor 2 sensitizes the capsaicin receptor transient receptor potential vanilloid receptor 1 to induce hyperalgesia. J. Neurosci. Off. J. Soc. Neurosci. 2004, 24, 4300-4312. [CrossRef]

32. Woo, Y.C.; Park, S.S.; Subieta, A.R.; Brennan, T.J. Changes in tissue $\mathrm{pH}$ and temperature after incision indicate acidosis may contribute to postoperative pain. Anesthesiology 2004, 101, 468-475. [CrossRef] [PubMed]

33. Wu, C.; Boustany, L.; Liang, H.; Brennan, T.J. Nerve growth factor expression after plantar incision in the rat. Anesthesiology 2007, 107, 128-135. [CrossRef] [PubMed]

34. Wu, C.; Erickson, M.A.; Xu, J.; Wild, K.D.; Brennan, T.J. Expression profile of nerve growth factor after muscle incision in the rat. Anesthesiology 2009, 110, 140-149. [CrossRef] [PubMed]

35. Carvalho, B.; Clark, D.J.; Yeomans, D.; Angst, M.S. Collecting and measuring nociceptive and inflammatory mediators in surgical wounds. J. Vis. Exp. 2008. [CrossRef] 
36. Kroin, J.S.; Buvanendran, A.; Watts, D.E.; Saha, C.; Tuman, K.J. Upregulation of cerebrospinal fluid and peripheral prostaglandin E2 in a rat postoperative pain model. Anesth. Analg. 2006, 103, 334-343, table of contents. [CrossRef]

37. Brennan, T.J.; Zahn, P.K.; Pogatzki-Zahn, E.M. Mechanisms of incisional pain. Anesthesiol Clin. N. Am. 2005, 23, 1-20. [CrossRef]

38. Hoogerwerf, W.A.; Zou, L.; Shenoy, M.; Sun, D.; Micci, M.A.; Lee-Hellmich, H.; Xiao, S.Y.; Winston, J.H.; Pasricha, P.J. The proteinase-activated receptor 2 is involved in nociception. J. Neurosci. Off. J. Soc. Neurosci. 2001, 21, 9036-9042. [CrossRef]

39. Zhao, P.; Lieu, T.; Barlow, N.; Sostegni, S.; Haerteis, S.; Korbmacher, C.; Liedtke, W.; Jimenez-Vargas, N.N.; Vanner, S.J.; Bunnett, N.W. Neutrophil Elastase Activates Protease-activated Receptor-2 (PAR2) and Transient Receptor Potential Vanilloid 4 (TRPV4) to Cause Inflammation and Pain. J. Biol. Chem. 2015, 290, 13875-13887. [CrossRef]

40. Poole, D.P.; Amadesi, S.; Veldhuis, N.A.; Abogadie, F.C.; Lieu, T.; Darby, W.; Liedtke, W.; Lew, M.J.; McIntyre, P.; Bunnett, N.W. Protease-activated receptor 2 (PAR2) protein and transient receptor potential vanilloid 4 (TRPV4) protein coupling is required for sustained inflammatory signaling. J. Biol. Chem. 2013, 288, 5790-5802. [CrossRef]

41. Mrozkova, P.; Palecek, J.; Spicarova, D. The role of protease-activated receptor type 2 in nociceptive signaling and pain. Physiol. Res. 2016, 65, 357-367. [CrossRef]

42. Spicarova, D.; Palecek, J. The role of spinal cord vanilloid (TRPV1) receptors in pain modulation. Physiol. Res. 2008, 57 (Suppl. 3), S69-S77. [PubMed]

43. Spicarova, D.; Adamek, P.; Kalynovska, N.; Mrozkova, P.; Palecek, J. TRPV1 receptor inhibition decreases CCL2-induced hyperalgesia. Neuropharmacology 2014, 81, 75-84. [CrossRef] [PubMed] 